\title{
Pharmacokinetic Drug-Drug Interactions of Mood Stabilizers and Risperidone in Patients Under Combined Treatment
}

\author{
Georgios Schoretsanitis, MD, *† Ekkehard Haen, MD, PhD, $\neq$ Gerhard Gründer, MD, * \\ Benedikt Stegmann, PhD, $\neq$ Koen R.J. Schruers, MD, PhD, $\$$ Christoph Hiemke, PhD, // \\ Sarah E. Lammertz, PhD, * and Michael Paulzen, MD*
}

\begin{abstract}
Background: The combination of anticonvulsant mood stabilizers with antipsychotic drugs may lead to clinically relevant drug-drug interactions. The objective of the study was to identify pharmacokinetic interactions of different mood stabilizers on the metabolism of risperidone (RIS) under natural conditions.

Methods: A large therapeutic drug monitoring database containing plasma concentrations of RIS and its metabolite 9-hydroxy-RIS (9-OH-RIS) of 1,584 adult patients was analyzed. Four groups $(n=1,072)$ were compared: a control group without a potentially cytochrome interacting comedication $\left(\mathrm{R}_{0}, \mathrm{n}=852\right)$, a group comedicated with valproate $(\mathrm{VPA})\left(\mathrm{R}_{\mathrm{VPA}}, \mathrm{n}=153\right)$, a group comedicated with lamotrigine (LMT) $\left(\mathrm{R}_{\mathrm{LMT}}, \mathrm{n}=46\right)$, and a group under concomitant medication with carbamazepine $(\mathrm{CBZ})\left(\mathrm{R}_{\mathrm{CBZ}}, \mathrm{n}=21\right)$. Dose-adjusted plasma concentrations (C/D ratio) for RIS, 9-OH-RIS and active moiety (AM) (RIS + 9-OH-RIS), as well as metabolic ratios (RIS/9-OH-RIS) were computed.
\end{abstract}

Results: Groups did not differ with regard to the daily dosage $(P=0.46)$. Differences were detected for the distributions of the C/D ratios for RIS, 9-OH-RIS and $\operatorname{AM}(P=0.003, P<0.001$ and $P<0.001$, respectively). Differences remained significant after conducting a Bonferroni correction $(P=0.0125)$. Pairwise comparisons of the concomitant medication groups with the control group revealed significant differences; RIS C/D ratios were significantly higher in the VPA and the LMT group than in the control group $(P=0.013 ; P=0.021)$. However, these differences did not remain significant after Bonferroni correction. In contrast, CBZ-treated patients showed lower dose-adjusted plasma concentrations of 9-OH-RIS $(P<0.001)$ as well as the AM $(P<0.001)$ than the control group; this difference survived the Bonferroni correction.

Conclusions: The data give evidence for pharmacokinetic interactions between RIS and different anticonvulsant mood stabilizers. Carbamazepine decreased serum concentrations of 9-OH-RIS and the AM when compared with the control group. In case of VPA and LMT, findings were less significant; hints for a weak RIS metabolism inhibition by LMT of unclear clinical significance were found.

Key Words: therapeutic drug monitoring, risperidone, carbamazepine, valproic acid, lamotrigine cytochrome $\mathrm{P} 450$, interaction, pharmacokinetics

(J Clin Psychopharmacol 2016;36: 00-00)

From the *Departmentof Psychiatry, Psychotherapy and Psychosomatics, RWTH Aachen University and JARA - Translational Brain Medicine, Aachen, Germany; †University Hospital of Psychiatry, Bern, Switzerland; †Clinical Pharmacology, Department of Psychiatry and Psychotherapy and Department of Pharmacology and Toxicology, University of Regensburg, Regensburg, Germany; §Faculty of Health, Medicine and Life Sciences, School for Mental Health and Neuroscience, Maastricht University, Maastricht, the Netherlands; ||Department of Psychiatry and Psychotherapy and Institute of Clinical Chemistry and Laboratory Medicine, University Medical Center of Mainz, Germany.

Received December 18, 2015; accepted after revision September 6, 2016.

Reprints: Michael Paulzen, MD, Department of Psychiatry, Psychotherapy and

Psychosomatics, and JARA - Translational Brain Medicine, RWTH

Aachen University, Pauwelsstr. 30, 52074 Aachen, Germany (e-mail:

mpaulzen@ukaachen.de).

Copyright (C) 2016 Wolters Kluwer Health, Inc. All rights reserved.

ISSN: 0271-0749

DOI: 10.1097/JCP.0000000000000601
G uidelines worldwide recommend the coadministration of second-generation antipsychotics as augmentative therapy to mood stabilizers or vice versa in the treatment of bipolar disorders, especially for the treatment of manic episodes. ${ }^{1-3}$ Acceptance of this strategy which promises better efficacy ${ }^{4}$ despite a higher potential of adverse events ${ }^{5,6}$ is reflected in increasing prescription trends. ${ }^{7}$ Many pharmacokinetic drug-drug interactions (DDIs) have been identified by therapeutic drug monitoring (TDM) as TDM databases enable evaluation of pharmacokinetic DDIs in a representative population to get an insight into the safety and tolerability of combined psychopharmacological treatment. Pharmacokinetic interactions in phase 1 drug metabolism, such as inhibition or induction of cytochrome P450 (CYP) activity, are the most important mechanisms of clinical DDIs. Combined pharmacotherapy can lead to desired effects, attenuated effects, and undesired harmful effects or even to serious adverse reactions. Even in complex clinical cases, valid interaction checks are still difficult, especially regarding the clinical relevance of a potential interaction risk. ${ }^{8}$ Most of the psychotropic drugs are metabolized extensively via CYP enzymes ${ }^{9}$ just like certain nonpsychiatric drugs, such as quinidine, terbinafine, or amiodarone, which are classified as strong, moderate or weak inhibitors of CYP2D6 according to the Food and Drug Administration (FDA) classification of in vivo inhibitors of CYP2D6, whereas no inducer of CYP2D6 has been identified. ${ }^{10}$ On the other hand, several CYP3A4 inhibitors (eg, clarithromycin, ketoconazole, and others) as well as inducers (eg, rifampin or carbamazepine [CBZ]) have been identified. ${ }^{10}$ In recent times, the appreciation for CYP-mediated DDI and the understanding of pharmacokinetic DDI has increased, and several tables of drugs as substrates, inhibitors, and inducers of drug-metabolizing CYP enzymes have been published. ${ }^{10,11}$ To understand the clinical relevance of DDIs for adverse drug reactions, the concept of a therapeutic window is important. ${ }^{12}$ Based on TDM ranges, risperidone (RIS) may be a narrow therapeutic window drug which — after adding an inhibitor or discontinuing an inducer - may be particularly prone to cause adverse drug reactions. ${ }^{13}$ However, any assessment regarding the magnitude of DDIs remains uncertain with regard to the great amount of never or scantily investigated drugs that might be cytochrome blockers or inducers.

Risperidone is a second-generation antipsychotic with antagonistic properties at serotonin $5-\mathrm{HT}_{2}$ and dopamine- $\mathrm{D}_{2}$ receptors. ${ }^{14}$ Risperidone has been used effectively in the treatment of schizophrenia and a broad spectrum of other psychiatric diseases. ${ }^{15}$ Risperidone is mainly metabolized via CYP2D6, and the main active metabolite is 9-hydroxy-RIS (9-OH-RIS, paliperidone). The socalled metabolic ratio (RIS/9-OH-RIS) can be used as a CYP2D6 phenotyping index ${ }^{16}$ that is influenced by CYP2D6 polymorphism and CYP2D6 inhibitors. ${ }^{17} \mathrm{~A}$ ratio above 1 (RIS/9-OH-RIS) $>1$ ) is an index of poor CYP2D6 metabolism. ${ }^{18}$ Differences in $5-\mathrm{HT}_{2 \mathrm{~A}} / \mathrm{D}_{2}$ binding ratios and the presence of a hydroxyl-group in the 9-OHRIS molecule increasing hydrophilicity might explain slightly differential pharmacological effects between 9-OH-RIS and the parent compound. ${ }^{19}$ In vitro findings have revealed that CYP3A4 and 
CYP3A5 might also be involved in the metabolism of RIS ${ }^{20-22}$ and possibly of 9-OH-RIS, a finding that was confirmed in clinical settings using powerful inducers and inhibitors of CYP3A showing decreased plasma concentrations of both, RIS and 9-OH-RIS, when CBZ was added. $^{23,24}$ Clinicians consider the combined concentration of RIS + 9-OH-RIS (active moiety [AM]) as the most relevant measure and according to the Arbeitsgemeinschaft für Neuropharmakologie und Pharmakopsychiatrie consensus guidelines, a therapeutic reference range is suggested as 20 to $60 \mathrm{ng} / \mathrm{mL}$ for the AM. ${ }^{11}$

Valproate (VPA) is an anticonvulsant drug that has been established as a mood stabilizer with pronounced antimanic effects as well as an antiaggressive agent. ${ }^{25,26}$ Its major metabolic pathways comprise, among others, an autoinductive $\beta$-oxidation and glucuronidation. ${ }^{27}$ Additionally, VPA is a substrate of CYP2C9 and $\mathrm{CYP} 2 \mathrm{C} 19^{28}$ and exerts a possible inhibitory effect on several enzyme systems including CYP2C9, CYP2C19, and uridine 5-diphospho-glucuronosyltransferase (UGT) ${ }^{29,30}$ whereas potential inhibitory effects on CYP3A4 seem to be without clinical significance so far. However, the mechanism and the clinical significance of possible interactions have not been fully elucidated and different pharmacokinetic effects on concomitantly applied have been shown, for example, acting simultaneously as an inhibitor but also as an inducer of clozapine and olanzapine metabolism. ${ }^{31}$ A recent study indicates that VPA might inhibit 9-OHRIS (paliperidone) metabolism increasing its oral bioavailability. ${ }^{32}$

Carbamazepine is frequently prescribed as a mood stabilizer or for mood and aggression control in patients with bipolar disorders and schizophrenia spectrum disorders. ${ }^{33}$ Carbamazepine is metabolized by CYP3A4/5 and CYP2C8, whereas some data imply the involvement of UGTs as well. ${ }^{34,35}$ Its primary metabolite, CBZ 10,11-epoxide, possesses anticonvulsant activity similar to the parent compound. There has been considerable evidence regarding the pharmacokinetic profile of CBZ and especially its DDI with other antiepileptic drugs, illustrating a major inducing effect of CBZ on CYP3A4 as well as similar effects on activities of other CYP isoenzymes, including CYP2A6, CYP2C9, CYP2C19, CYP2B6, CYP1A2, UGTs, and P-glycoprotein. ${ }^{31,36,37}$ Moreover, Yatham and colleagues ${ }^{38}$ detected $40 \%$ lower plasma concentrations of RIS AM values in CBZ-treated patients compared with patients receiving RIS and VPA or lithium. These findings are in agreement with data from a small clinical sample reporting a CYP3A4-mediated interaction between CBZ and RIS, leading to decreased plasma levels of the parent compound RIS. ${ }^{39}$ Hence, due to its complex pharmacokinetics, CBZ can substantially affect the disposition of a variety of psychotropic agents.

Lamotrigine (LMT) is an antiepileptic drug that is recommended in bipolar disorder maintenance when depression is prominent. ${ }^{40}$ Clinical data support its adjunctive prescription in managing treatment-resistant positive symptoms in schizophrenic patients. ${ }^{41}$ Lamotrigine is metabolized primarily by glucuronidation. ${ }^{42}$ Uridine-diphosphate-glucuronosyltransferase 1A4 (UGT1A4) catalyzes $90 \%$ of LMT conjugation and the major metabolite, LMT-2-N-glucuronide, is excreted via the kidneys. ${ }^{43}$ Lamotrigine is known to induce its own metabolism (via UGT1A4), but so far there is no evidence that LMT interacts with drugs that are metabolized via the CYP enzyme system.

The aim of the study was to investigate whether VPA or CBZ have the potential to interact with the CYP2D6 or CYP3A4 mediated RIS metabolism reflected in changes of plasma concentrations of RIS; its active metabolite 9-OH-RIS or the AM, RIS + 9-OHRIS. Furthermore, we were interested in a potential interaction between LMT and RIS because this interaction has been described to occur with increasing plasma concentrations of RIS and 9-OHRIS, though an explanation for this effect is thus far lacking. ${ }^{44}$

\section{MATERIALS AND METHODS}

The study was conducted as a cooperation between the Department of Psychiatry, Psychotherapy, and Psychosomatics of RWTH Aachen University Hospital, Germany, and the Department of Psychiatry and Psychotherapy at the University of Regensburg, Germany. Konzentrationsbestimmung von Psychopharmaka, a large TDM database $\mathrm{s}^{45,46}$ containing thousands of plasma concentrations of RIS and 9-OH-RIS was the source of our data. The TDM databases are a valuable source to promote the safety and tolerability of pharmacotherapy in a clinical setting. ${ }^{47,48}$ In the case of multiple available plasma concentrations for 1 single patient, only the most recent value was included in the analysis. After adjusting the database for multiple values, and after excluding patients with depot formulations, RIS and 9-OH-RIS plasma concentrations were available for 1,584 adult inpatients and outpatients who had been treated with RIS for different reasons. Data collection took place between 2006 and 2015 as part of the clinical routine in different institutions as part of the Arbeitsgemeinschaft Arzneimittelsicherheit bei psychischen Erkrankungen, a cooperation for drug safety in the treatment of psychiatric diseases (for details, www.amuep-agate.de). Retrospective analysis of clinical data for this study was in accordance with the local regulatory authority.

We considered 4 groups; a group of patients receiving RIS as an oral formulation (control group, $\mathrm{R}_{0}$ ), a group comedicated with VPA $\left(\mathrm{R}_{\mathrm{VPA}}\right)$, a group comedicated with LMT $\left(\mathrm{R}_{\mathrm{LMT}}\right)$, and a group with $\mathrm{CBZ}\left(\mathrm{R}_{\mathrm{CBZ}}\right)$. No matching processes for age, diagnoses, severity of illness, length, or onset of illness were undertaken. The control group consists of patients without a comedication with CYP2D6 or CYP3A4 inhibitory or CYP3A4 inducing properties according to current knowledge. ${ }^{10,11}$

\section{Quantification of RIS and 9-OH-RIS}

Blood was drawn just before drug administration (trough concentration) at steady state ( $>5$ elimination half-lives under the same drug dose). Risperidone and 9-OH-RIS concentrations were determined by high performance liquid chromatography (HPLC) with ultraviolet (HPLC/UV) detection (HPLC/UV). ${ }^{49,50}$ The UV absorption wavelength was set to $235 \mathrm{~nm}$ for quantification. The Dionex DAD detector allowed simultaneous documentation of a second signal at $280 \mathrm{~nm}$, which was used to double-check the purity of the signal at $235 \mathrm{~nm}$. The method was validated according to DIN 32645 (Deutsche Industrie Norm 32645, described in guidelines of GTFCh (Society of Toxicology and Forensic Chemistry) in consideration of International Organization for Standardization $5725,{ }^{51}$ US FDA guidelines, ${ }^{52}$ and International Conference on Harmonization requirements. ${ }^{53}$ The laboratory regularly runs internal quality controls and participates in external quality assessment schemes by INSTAND (Düsseldorf, Germany, www. instandev.de). The limit of detection, defined as signal to noise ratio of 3:1, was $5 \mathrm{ng} / \mathrm{mL}$ for both RIS and 9-OH-RIS. The interday precision, determined as duplicates on 3 different days at $5 \mathrm{ng} / \mathrm{mL}$ was $5.56 \%$ of mean and $5.21 \%$ of mean, respectively. Usually, twice the limit of detection is accepted as limit of quantification. However, the analytical process using HPLC with UV (HPLC/UV) detection also detects both RIS and 9-OH-RIS values below the limit of quantification, and we decided to include these defined values into our analysis because they were true values above 0 .

\section{Statistical Analysis}

Medians and the distributions of the plasma concentrations of RIS, 9-OH-RIS, and the AM (RIS + 9-OH-RIS) were compared. Furthermore, we compared the ratio of RIS/9-OH-RIS, a parameter that has been demonstrated to be an excellent measure of the CYP2D6 activity. ${ }^{16,54}$ We also calculated the dose-adjusted 
TABLE 1. Patients' Demographic Characteristics

\begin{tabular}{lccccc}
\hline Group & & & \multicolumn{2}{c}{ Sex } & DD RIS, mg/d \\
\cline { 4 - 6 } & Number & Age, $\mathbf{y}$ & \% Females & \% Males & Median (Range) \\
\hline Valproate $\left(\mathrm{R}_{\mathrm{VPA}}\right)$ & 153 & $41.7(18-83)$ & 44.4 & 55.6 & $4.0(1.00-10.0)$ \\
Lamotrigine $\left(\mathrm{R}_{\mathrm{LMT}}\right)$ & 46 & $46.5(20-72)$ & 60.9 & 39.1 & $4.0(1.0-10.0)$ \\
Carbamazepine $\left(\mathrm{R}_{\mathrm{CBZ}}\right)$ & 21 & $49.85(23-83)$ & 71.4 & 28.6 & $6.0(1.0-9.0)$ \\
Control $\left(\mathrm{R}_{0}\right)$ & 852 & $41.25(18-87)$ & 44.2 & 55.8 & $4.0(1.00-10.0)$ \\
\hline
\end{tabular}

DD indicates daily dose.

plasma concentration (C/D ratio) for RIS, 9-OH-RIS and AM (RIS + 9-OH-RIS), as the ratio of the plasma concentration of RIS, 9-OH-RIS or RIS + 9-OH-RIS divided by the daily dose of RIS (in $[(\mathrm{ng} / \mathrm{mL}) / \mathrm{mg}])$. As the data were not normally distributed, a nonparametrical Kruskal-Wallis test and a median test, both with a significance level of 0.05 , were conducted. For each comparison of the 3 comedicated groups $\left(\mathrm{R}_{\mathrm{VPA}}, \mathrm{R}_{\mathrm{LMT}}, \mathrm{R}_{\mathrm{CBZ}}\right)$ with the control group $\left(\mathrm{R}_{0}\right)$, we used the Mann-Whitney $U$ test with the same significance level. Furthermore, we conducted a Bonferroni correction, setting a significance cutoff at 0.0125 . Statistical analysis was carried out using IBM SPSS Statistics version 18.0 (IBM $\mathrm{GmbH}$, Ehningen, Germany).

\section{RESULTS}

One thousand seventy-two of 1,584 patients were eligible for analysis (no other confounding comedication according to the FDA classification of in vivo inhibitors of CYP enzymes ${ }^{10}$ ) and were assigned to the 4 groups, $R_{0}(n=852), R_{V P A}(n=153), R_{L M T}$ $(n=46)$, and $R_{C B Z}(n=21)$. The demographic data of these patients are summarized in Table 1.

The median plasma concentrations $(\mathrm{ng} / \mathrm{mL})$ of RIS, 9-OHRIS, the AM (RIS + 9-OH-RIS), as well as the metabolic ratios (RIS/9-OH-RIS) are displayed in Table 2 and in Fig. 1.

Table 3 shows the C/D ([ng/mL]/mg) for RIS, 9-OH-RIS, and RIS + 9-OH-RIS.

Because of the skewness of the distribution, comparisons were based upon the Kruskal-Wallis test. No differences were found regarding the distribution and the medians of daily dosage of RIS between the groups ( $P=0.46$ and $P=0.086)\left(\mathrm{R}_{0}\right.$ : mean, $4.34 \mathrm{mg} / \mathrm{d}$; SD, 2.03; $\mathrm{R}_{\mathrm{VPA}}$ : mean, $4.43 \mathrm{mg} / \mathrm{d}$; SD, 2.21; $\mathrm{R}_{\mathrm{LMT}}$ : mean, $4.51 \mathrm{mg} / \mathrm{d}$; SD, 2.19; and $\mathrm{R}_{\mathrm{CBZ}}$ : mean, $5.07 \mathrm{mg} / \mathrm{d}$; $\mathrm{SD}$, 2.44). The comparison of the distribution (subscript indices ' $D$ ') and the medians (subscript indices ' $\mathrm{M}$ ') of the plasma concentrations of RIS, 9-OH-RIS, and the AM (RIS + 9-OH-RIS) between the 4 groups yielded significant differences in all cases RIS $_{\mathrm{D}}$, $P=0.006$; 9-0H-RIS $\mathrm{D}, P=0.008$; and RIS $+9-\mathrm{OH}-\mathrm{RIS}_{\mathrm{D}}$, $P=0.004 ;$ RIS $_{\mathrm{M}}, P=0.021 ; 9-0 \mathrm{H}-\mathrm{RIS}_{\mathrm{M}}, P=0.012$ and RIS $+9-$ $\left.\mathrm{OH}-\mathrm{RIS}_{\mathrm{M}}, P=0.002\right)$. Similarly, the distributions of the doseadjusted plasma concentrations, $\mathrm{C} / \mathrm{D}$ ratios, showed significant differences between the groups $\left(\mathrm{RIS}_{\mathrm{D}} \mathrm{C} / \mathrm{D}\right.$ ratio, $P=0.003$; 9-OH-RIS $\mathrm{D}_{\mathrm{D}} \mathrm{C} / \mathrm{D}$ ratio, $P<0.001$; and RIS $+9-\mathrm{OH}-\mathrm{RIS}_{\mathrm{D}} \mathrm{C} / \mathrm{D}$ ratio, $P<0.001$ ). When comparing medians of these parameters, we detected significant differences between the groups for 9-OH-RIS $\mathrm{C} / \mathrm{D}$ and RIS + 9-OH-RIS C/D but not for RIS C/D (9-OH-RIS $\mathrm{C} / \mathrm{D}$ ratio, $P<0.001$; RIS $+9-\mathrm{OH}-\mathrm{RIS}_{\mathrm{M}} \mathrm{C} / \mathrm{D}$ ratio, $P=0.002$; RIS $_{\mathrm{D}} \mathrm{C} / \mathrm{D}$ ratio, $\left.P=0.108\right)$. Significant differences were also detected between the distributions, but not the medians of RIS/9OH-RIS ratios of 4 groups $(P=0.004$ and $P=0.051)$.

To eliminate type 1 errors, we chose to apply a Bonferroni correction adjusting our initial $P$ value and setting a cutoff of 0.0125 . Differences between the distributions and the medians remained significant with the exception of the differences between median plasma concentrations of RIS (Fig. 2).

To control for significant differences between pairs of groups ( $\mathrm{R}_{0}$ vs $\mathrm{R}_{\mathrm{VPA}}, \mathrm{R}_{0}$ vs $\mathrm{R}_{\mathrm{LMT}}$, and $\mathrm{R}_{0}$ vs $\mathrm{R}_{\mathrm{CBZ}}$ ), a Mann-Whitney $U$ test was used. Patients that were comedicated with VPA showed significantly higher plasma concentrations and a higher RIS C/D ratio compared with the control group $(P=0.016$ and $P=0.013)$, whereas plasma concentrations and 9-OH-RIS C/D ratio $(P=0.869$ and $P=0.585)$ as well as plasma concentrations and RIS $+9-\mathrm{OH}-\mathrm{RIS} \mathrm{C} / \mathrm{D}$ ratio $(P=0.221$ and $P=0.111)$ showed no significant differences. However, the metabolic ratios (RIS/9-OH-RIS) were significantly different, with higher values in the VPA group $(P=0.017)$. After applying the Bonferroni correction, none of the differences remained significant.

TABLE 2. Median (and Range) Plasma Concentrations and Metabolic Ratios of Risperidone in the Study Groups

\begin{tabular}{lcccc}
\hline Group & RIS, ng/mL & 9-OH-RIS, $\mathbf{n g} / \mathbf{m L}$ & RIS + 9-OH-RIS, ng/mL & RIS/9-OH-RIS \\
\hline Valproate $\left(\mathrm{R}_{\mathrm{VPA}}\right)$ & $7.0^{*}(0.1-252.0)$ & $16.0(1.2-147.0)$ & $27.0(1.7-255.7)$ & $0.363^{*}(0.009-68.1)$ \\
Lamotrigine $\left(\mathrm{R}_{\mathrm{LMT}}\right)$ & $6.4^{\dagger}(0.2-60.9)$ & $15.5(2.0-83.5)$ & $23.5(8.0-144.4)$ & $0.525^{\dagger}(0.008-27.0)$ \\
Carbamazepine $\left(\mathrm{R}_{\mathrm{CBZ}}\right)$ & $4.0(0.2-42.0)$ & $9.6^{\ddagger}(1.9-55.0)$ & $14.8^{\ddagger}(3.3-57.9)$ & $0.333(0.052-5.25)$ \\
Control $\left(\mathrm{R}_{0}\right)$ & $4.4(0.1-224.0)$ & $17.0(0.3-196.5)$ & $24.0(1.8-264.0)$ & $0.262(0.003-23.68)$ \\
\hline
\end{tabular}

*Plasma concentration values for RIS and metabolic ratios in the $\mathrm{R}_{\mathrm{VPA}}$ group were significantly higher compared with the control group (uncorrected $P=0.016$ and $P=0.017$ for Mann-Whitney $U$ Test).

†Plasma concentration values for RIS and metabolic ratios in the $\mathrm{R}_{\mathrm{LMT}}$ group were significantly higher compared with the control group (uncorrected $P=0.016$ and $P=0.004$ for Mann-Whitney $U$ Test).

FPlasma concentration values for 9-OH-RIS and RIS + 9-OH-RIS in the $\mathrm{R}_{\mathrm{CBZ}}$ group were significantly lower than in the control group (uncorrected $P=0.001$ and $P=0.001$ for Mann-Whitney $U$ Test). 

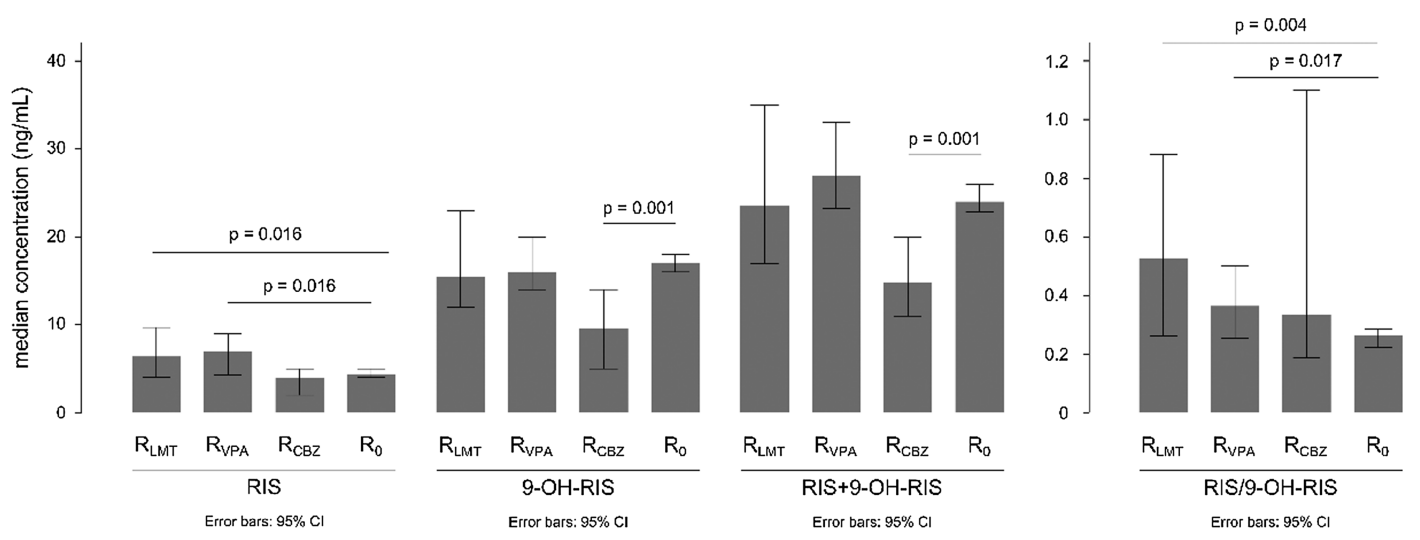

FIGURE 1. Median plasma concentrations of RIS, 9-OH-RIS and active moiety as well as the metabolic ratios (RIS/9-OH-RIS) in the 4 different study groups. $\mathrm{R}_{\mathrm{LMT}}=$ lamotriginegroup; $\mathrm{R}_{\mathrm{VPA}}=$ valproate group; $\mathrm{R}_{\mathrm{CBZ}}=$ carbamazepine group; $\mathrm{R}_{0}=$ control group. $\mathrm{Cl}=$ confidence interval. C/D for RIS, 9-OH-RIS and RIS + 9-OH-RIS in [(ng/ml)/mg]. Metabolic ratio (RIS/9-OH-RIS) without unit.

The Mann-Whitney $U$ test revealed similar patterns of distribution differences when comparing the LMT group versus the control group. Plasma concentrations and dose-adjusted plasma concentrations of the parent compound, RIS, differed between the groups, with LMT patients showing higher values $(P=0.016$ and $P=0.021)$. Differences failed to reach significance levels in case of the AM $(P=0.732$ for RIS $+9-$ OH-RIS and $P=0.745$ for RIS + 9-OH-RIS C/D ratio) as well as in the case of active metabolite $(P=0.552$ for $9-\mathrm{OH}-\mathrm{RIS}$ and $P=0.182$ for $9-\mathrm{OH}-\mathrm{RIS} \mathrm{C} / \mathrm{D}$ ratio) Metabolic ratio (RIS/9-OH-RIS) values were higher in the LMT group $(P=0.004)$ and remained significant after the Bonferroni correction, whereas the others did not.

The coadministration of CBZ led to completely different findings. Comparing the $\mathrm{CBZ}$ versus the control group yielded no significant differences regarding RIS $(P=0.29$ for RIS and $P=0.096$ for RIS C/D ratio), and the metabolic ratios did not differ between the 2 groups $(P=0.253)$. However, both the plasma concentration of the active metabolite as well as the AM were significantly lower in the CBZ group $(P=0.001$ for 9-OH-RIS and $P<0.001$ for 9-OH-RIS C/D ratio, $P=0.001$ for RIS $+9-\mathrm{OH}-$ RIS, and $P<0.001$ for RIS + 9-OH-RIS C/D ratio). The Bonferroni correction did not change results as all differences remained significant.

Further analysis included the distribution pattern, C/D and metabolic ratios for the study groups. In the CBZ group, only 1 patient had a 9-OH-RIS C/D ratio higher than the median, whereas the rest of the group $(n=20)$ showed a 9-OH-RIS C/D ratio below the median. Similarly striking are the findings regarding the AM C/D ratios, with 2 patients in the CBZ group showing RIS + 9-OH-RIS C/D values above the median. Although considering this distribution pattern differences, it should be kept in mind that the median 9-OH-RIS and AM C/D ratios were significantly lower in the CBZ group compared with all 3 groups $(P<0.001$ and $P=0.002)$. Considering the distribution of the RIS C/D values of the 4 groups, patients with a coadministration of CBZ tend to show median values below the median, whereas in the 2 other groups and in the control group, the distributions were more balanced.

Considering the effects of the other 2 investigated mood stabilizers (VPA and LMT), findings reached statistical significance regarding RIS plasma concentrations and $\mathrm{C} / \mathrm{D}$ values, but were not substantially reflected in differences in the AM $\left(\mathrm{R}_{\mathrm{VPA}}\right.$ vs $\mathrm{R}_{0}$ : $P=0.221$ for RIS + 9-OH-RIS and $P=0.111$ for RIS + 9-OHRIS C/D ratio; $\mathrm{R}_{\mathrm{LMT}}$ vs $\mathrm{R}_{0}: P=0.732$ for RIS $+9-\mathrm{OH}-\mathrm{RIS}$ and $P=0.745$ for RIS $+9-\mathrm{OH}-\mathrm{RIS} \mathrm{C} / \mathrm{D}$ ratio).

\section{DISCUSSION}

The augmentation of an antipsychotic pharmacotherapy with an anticonvulsant as a mood stabilizer is a very common psychopharmacological practice. However, by adding an anticonvulsant to an ongoing antipsychotic treatment, the risk of pharmacokinetic DDIs is increasing. For predicting potential DDIs, knowledge about pharmacokinetic interactions, predominantly involving the CYP system, is of high value. For a better understanding of potentially occurring DDIs in complex multiple drug therapy settings, data from TDM surveys are an ideal source for identification and quantification of risks. ${ }^{55,56}$

In our naturalistic sample, patients on a stable dose with RIS in the control group and patients that were comedicated with VPA,

TABLE 3. Median C/D of Risperidone in the Different Groups

\begin{tabular}{lccc}
\hline Group & RIS C/D ratio $[(\mathbf{n g} / \mathbf{m l}) / \mathbf{m g}]$ & 9-OH-RIS C/D ratio [(ng/ml)/mg] & RIS + 9-OH-RIS C/D ratio [(ng/mI)/mg] \\
\hline Valproate $\left(\mathrm{R}_{\mathrm{VPA}}\right)$ & $1.77^{*}(0.03-63.0)$ & $4.00(0.27-23.33)$ & $6.66(0.38-63.93)$ \\
Lamotrigine $\left(\mathrm{R}_{\mathrm{LMT}}\right)$ & $1.5^{\dagger}(0.03-27.0)$ & $3.83(0.31-27.83)$ & $6.02(1.3-48.13)$ \\
Carbamazepine $\left(\mathrm{R}_{\mathrm{CBZ}}\right)$ & $0.66(0.13-10.5)$ & $2.00^{\ddagger}(0.48-6.88)$ & $2.62^{\ddagger}(1.7-12.5)$ \\
Control $\left(\mathrm{R}_{0}\right)$ & $1.16(0.02-74.67)$ & $4.33(0.08-42.0)$ & $6.29(0.5-88.0)$ \\
\hline
\end{tabular}

*C/D values for RIS in the $\mathrm{R}_{\mathrm{VPA}}$-group were significantly higher than in the control group (uncorrected $P=0.013$, for Mann-Whitney $U$ test).

${ }^{\dagger} \mathrm{C} / \mathrm{D}$ values for RIS in the $\mathrm{R}_{\mathrm{LMT}}$ group were significantly higher than in the control group (uncorrected $P=0.021$ for Mann-Whitney $U$ test).

${ }^{\star} \mathrm{C} / \mathrm{D}$ values for 9-OH-RIS and RIS $+9-\mathrm{OH}-\mathrm{RIS}$ in the $\mathrm{R}_{\mathrm{CBZ}}$ group were significantly lower than in the control group (uncorrected $P<0.001$ and $P<0.001$ for Mann-Whitney $U$ test). 


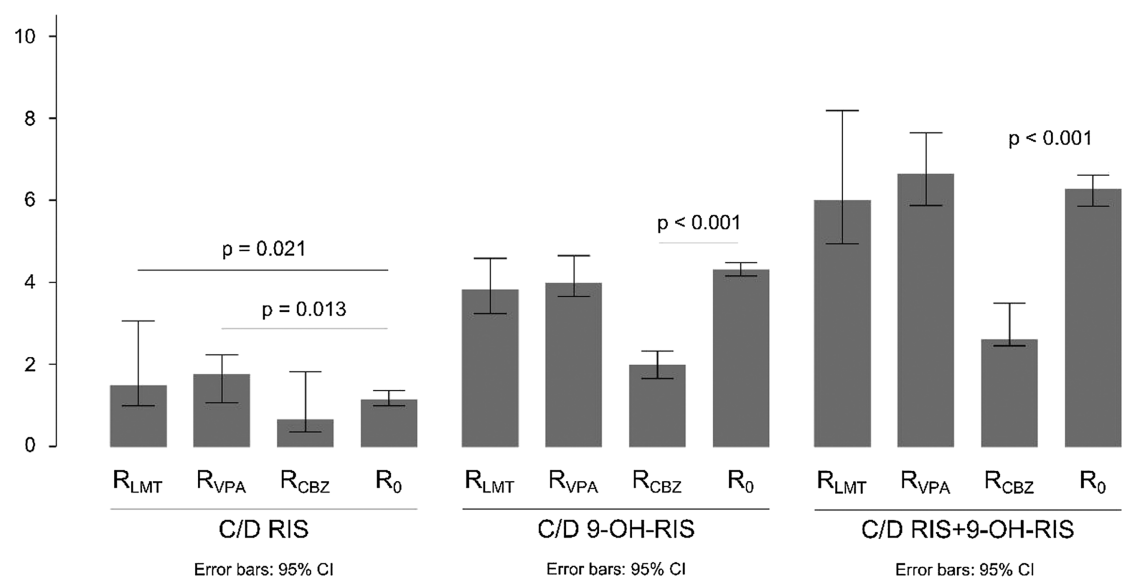

FIGURE 2. Median dose-adjusted plasma concentrations (C/D) for risperidone, 9-hydroxyrisperidone and active moiety in the different groups. $\mathrm{R}_{\mathrm{LMT}}=$ lamotrigine group; $\mathrm{R}_{\mathrm{VPA}}=$ valproate group; $\mathrm{R}_{\mathrm{CBZ}}=$ carbamazepine group; $\mathrm{R}_{0}=$ control group. C/D for RIS, 9-OH-RIS and $\mathrm{RIS}+9-\mathrm{OH}-\mathrm{RIS}$ in $[(\mathrm{ng} / \mathrm{ml}) / \mathrm{mg}]$.

LMT, or CBZ showed considerably different patterns of plasma concentrations of RIS, 9-OH-RIS, and AM as well as different patterns of dose-adjusted plasma concentrations (C/D).

The most impressive findings were seen in the CBZ group. Both the median plasma concentration and the dose-adjusted plasma concentration of 9-OH-RIS and AM were about 2-fold lower than in the control group, revealing results that are in line with correction factors for potent inducers, such as CBZ, as suggested by de Leon et al. ${ }^{4,31}$ The comparison of the plasma and the dose-adjusted plasma concentrations of RIS between the $\mathrm{CBZ}$ and the control group did not yield statistically significant differences. However, the numeric difference has to be taken into account, that is, in case of RIS C/D ratio, the median value for CBZ patients was $0.66(\mathrm{ng} / \mathrm{mL}) / \mathrm{mg}$, whereas it was $1.16(\mathrm{ng} /$ $\mathrm{mL} / \mathrm{mg}$ in the control group. Significantly lower concentrations might be due to an induction of the cytochrome mediated metabolism of RIS. Nonetheless, the inducing properties of CBZ are rather unlikely to be attributed to an enhanced 9-hydroxylation of RIS via CYP2D6 but might be the result of an induction of alternative metabolic pathways, such as the CYP3A4-mediated metabolism of RIS. Given that the plasma concentration of RIS did not show significant differences between the $\mathrm{CBZ}$ and the control group ( $P=0.383$ for RIS and $P=0.165$ for RIS C/D ratio), we were able to rule out the possibility that this prominent decrease may somehow be due to differences of plasma concentration of RIS.

Although the daily dose of RIS was not significantly different between the study groups, significantly lower plasma concentrations of 9-OH-RIS in the CBZ group contributed to a substantially lower concentration of the AM with potential clinical implications $(P=0.001$ for RIS + 9-OH-RIS and $P<0.001$ for RIS + 9-OHRIS C/D ratio), although we did not analyze clinical features due to our pharmacokinetic approach. Differences between patients that were comedicated with $\mathrm{CBZ}$ and those in the control-group remained significant after conducting a Bonferroni correction. Our observation is in accordance with previous small clinical studies and case reports that indicated an inducing effect of CBZ on the RIS metabolism. ${ }^{23,38,39,57}$ The clinical relevance of the alterations in RIS metabolism attributed to CBZ has been demonstrated by case reports, for example, an acute psychotic exacerbation after prescribing CBZ in a patient under RIS ${ }^{58}$ or the appearance of extrapyramidal symptoms (EPS) after withdrawing CBZ from the therapeutic regimen. ${ }^{59}$

Consequently, individualization of the doses or better the therapeutic plasma concentrations of antipsychotics, such as RIS, using TDM should be considered to maintain a favorable antipsychotic effect when CBZ is coadministered, or to avoid adverse reactions when a CYP-inducing comedication is stopped. ${ }^{60,61}$ Especially in the case of CBZ discontinuation, Punyawudho and colleagues ${ }^{62}$ estimated that enzyme induction should be reduced by about half after 3 days of discontinuation, by $75 \%$ after 7 days and a return to a normal status might be achieved after 2 weeks.

In terms of VPA, in vitro findings may be explained by inhibiting properties on CYP3A $4,{ }^{29}$ which is further supported by in vivo findings that showed inhibiting effects of VPA on the metabolism of quetiapine, a CYP3A4 substrate. ${ }^{63}$ A weak inhibition of the CYP3A4-mediated metabolism of RIS offers a plausible interpretation of our findings although the findings did not survive Bonferroni correction.

With regard to possible pharmacokinetic interactions between RIS and LMT, data are lacking so far. In our study, the RIS/9-OHRIS ratio in the LMT group was significantly higher, and although statistical significance of higher serum concentrations of the parent compound RIS did not survive Bonferroni correction, the RIS/9$\mathrm{OH}-\mathrm{RIS}$ ratio remained significant. A higher RIS/9-OH-RIS ratio in patients who are comedicated with LMT might be considered as a hint for a weak inhibiting effect on the CYP 3A4-mediated metabolism of RIS or for an inhibition of RIS metabolism that might not be limited to phase 1 metabolism. Only 1 single case report demonstrated enhanced RIS plasma concentrations after a concomitant therapy with $\mathrm{LMT}^{44}$ whereas findings from a small clinical sample did not report changes in RIS concentrations after coadministration of LMT. ${ }^{64}$ Literature data suggest UGT-mediated interactions of LMT with other psychotropic agents. ${ }^{64,65}$ Hence, it should be pointed out that a possible weak inhibitory effect of LMT on RIS metabolism - mediated by either cytochrome or UGT - might explain our findings but is unlikely to be of clinical significance.

Summarizing the findings, CYP3A4 seems to play a substantial role in the metabolism of RIS, consistent with in vitro findings that have illustrated an involvement of CYP3A4 in the metabolism of RIS. In the light of our findings any additionally prescribed comedication to an ongoing RIS treatment has to be critically evaluated regarding CYP2D6 inhibiting and/or CYP3A4 inhibiting or inducing properties. In case of CBZ, another explanation of the interaction pharmacology may be seen in its P-glycoprotein inducing properties, while RIS is a substrate of P-gp. So the combination of CBZ and RIS could lead to lower plasma concentrations of RIS and 9-OH-RIS as a consequence of a reduced absorption from the gastrointestinal tract. ${ }^{37}$ Regarding the interaction of VPA and LMT with RIS, findings were less clear with only 
a higher RIS/9-OH-RIS ratio for LMT surviving the adjustment of the significance cutoff. This might imply a mild inhibitory effect of LMT on RIS metabolism that clinicians should be aware of.

\section{Limitations}

Our sample comprised a large population of naturalistic observations and relies on retrospective data. A significant amount of clinical parameters (onset and duration of illness, adverse effects, comorbidities, duration of drug exposure) were not available. Furthermore, we could not control for potentially large individual variations in sampling time (although clinicians were asked to draw blood at trough level times) as a result of the clinical setting. This may have contributed to the pronounced interindividual variation in plasma concentrations and metabolic ratios. Other important limitations can be seen in the lack of information about CYP2D6 genotypes (PMs around 7\% of the sample) and lack of information on renal insufficiency as 9-OH-RIS is mainly eliminated via the kidneys. Independent of differences in clinical routine, a large interindividual variability in RIS and 9-OH-RIS concentrations has already been reported in the literature. ${ }^{66} \mathrm{In}$ the case of multiple plasma concentration determinations, we minimized the patient bias by including only the most recent analysis per patient. Regarding the analyses of the data, we observed differences in sample characteristics and sample size in the 4 patient groups; thus, the comparability of the study groups with the control group might be restricted.

Consequently, we avoided stratifying for age and sex despite the previously reported effect of both on RIS metabolism to evade perplexing our analysis. ${ }^{17,67}$ We chose not to reduce the control group taking into account the extent of the skewness of the sample distribution. Finally, statistical significance is heavily influenced by sample size, and our findings have to be considered keeping in mind the effect sizes.

\section{Clinical points}

- The combination of mood stabilizers with antipsychotic drugs is a common augmentation strategy but may lead to clinically relevant pharmacokinetic interactions.

- Evidence is provided for pharmacokinetic interactions between RIS and different mood stabilizers.

- CBZ provides the highest pharmacokinetic interaction potential with RIS but also valproic acid and LMT may lead to significant changes in plasma concentrations of RIS, its active metabolite and the AM.

\section{ACKNOWLEDGMENTS}

The authors thank Mrs Lisa Deuse, RWTH Aachen University, and A. C. Parker, M.D., Department of Obstetrics \& Gynecology, New York Hospital, Cornell Medical Centre, New York, NY (emeritus) for careful reading of this manuscript.

\section{AUTHOR DISCLOSURE INFORMATION}

Ekkehard Haen received speaker's or consultancy fees from the following pharmaceutical companies: Servier, Novartis, and Janssen-Cilag. He is managing director of Arbeitsgemeinschaft Arzneimittelsicherheit bei psychischen Erkrankungen, a nonprofit working group to improve drug safety and efficacy in the treatment of psychiatric diseases. He reports no conflict of interest with this publication. Christoph Hiemke has received speaker's or consultancy fees from the following pharmaceutical companies: AstraZeneca, Janssen-Cilag, Pfizer, Lilly and Servier. He is managing director of the psiac GmbH which provides an internet based DDI program for psychopharmacotherapy. He reports no conflict of interest with this publication. Gerhard Gründer has served as a consultant for Boehringer Ingelheim (Ingelheim, Germany), Cheplapharm (Greifswald, Germany), Eli Lilly (Indianapolis, Ind, USA), Lundbeck (Copenhagen, Denmark), Ono Pharmaceuticals (Osaka, Japan), Roche (Basel, Switzerland), Servier (Paris, France), and Takeda (Osaka, Japan). He has served on the speakers' bureau of Eli Lilly, Gedeon Richter (Budapest, Hungary), Janssen Cilag (Neuss, Germany), Lundbeck, Roche, Servier, and Trommsdorf (Aachen, Germany). He has received grant support from Boehringer Ingelheim and Roche. He is co-founder of Pharma Image GmbH (Düsseldorf, Germany) and Brainfoods UG (Selfkant, Germany). He reports no conflict of interest with this publication. Georgios Schoretsanitis received a grant from the bequest "in memory of Maria Zaoussi", State Scholarships Foundation, Greece for clinical research in Psychiatry for the academic year 2015-2016. All other authors declare no conflicts of interest as well. The research study did not receive funds or support from any source.

G.S., M.P., G.G., C.H., E.H., B.S., and K.R.J.S. participated in research design. G.S., M.P., S.E.L. performed data analysis. G.S., M.P., G.G., C.H., E.H., B.S., K.R.J.S., and S.E.L. wrote or contributed to the writing of the article.

\section{REFERENCES}

1. Kendall T, Morriss R, Mayo-Wilson E, et al. Assessment and management of bipolar disorder: summary of updated NICE guidance. BMJ. 2014; 349:g5673.

2. Grunze H, Vieta E, Goodwin GM, et al. The World Federation of Societies of Biological Psychiatry (WFSBP) guidelines for the biological treatment of bipolar disorders: update 2012 on the long-term treatment of bipolar disorder. World J Biol Psychiatry. 2013;14:154-219.

3. Yatham LN, Kennedy SH, Parikh SV, et al. Canadian Network for Mood and Anxiety Treatments (CANMAT) and International Society for Bipolar Disorders (ISBD) collaborative update of CANMAT guidelines for the management of patients with bipolar disorder: update 2013. Bipolar Disord. 2013;15:1-44.

4. de Leon J, Santoro V, D'Arrigo C, et al. Interactions between antiepileptics and second-generation antipsychotics. Expert Opin Drug Metab Toxicol. 2012;8:311-334

5. Buoli M, Serati M, Altamura AC. Is the combination of a mood stabilizer plus an antipsychotic more effective than mono-therapies in long-term treatment of bipolar disorder? A systematic review. J Affect Disord. 2014; 152-154:12-18

6. Leucht S, Helfer B, Dold M, et al. Carbamazepine for schizophrenia. Cochrane Database Syst Rev. 2014;5:CD001258.

7. Bjorklund L, Horsdal HT, Mors O, et al. Trends in the psychopharmacological treatment of bipolar disorder: a nationwide register-based study. Acta Neuropsychiatr. 2016;28:75-84.

8. Paulzen M, Eap CB, Gründer G, et al. Pharmacokinetic interaction between valproic acid, meropenem, and risperidone. J Clin Psychopharmacol. 2016; 36:90-92.

9. Stingl JC, Brockmöller J, Viviani R. Genetic variability of drug-metabolizing enzymes: the dual impact on psychiatric therapy and regulation of brain function. Mol Psychiatry. 2013;18:273-287.

10. US Food and Drug Administration. Drug development and drug interactions: table of substrates, inhibitors and inducers. 2014 [Available from: http:/www.fda.gov/Drugs/DevelopmentApprovalProcess/ DevelopmentResources/DrugInteractionsLabeling/ucm093664.htm. [Accessed 08/18/2016]

11. Hiemke C, Baumann P, Bergemann N, et al. AGNP Consensus Guidelines for Therapeutic Drug Monitoring in Psychiatry: update 2011. Pharmacopsychiatry. 2011;44:195-235.

12. de Leon J. The crucial role of the therapeutic window in understanding the clinical relevance of the poor versus the ultrarapid metabolizer phenotypes 
in subjects taking drugs metabolized by CYP2D6 or CYP2C19. J Clin Psychopharmacol. 2007;27:241-245.

13. Spina E, Hiemke C, de Leon J. Assessing drug-drug interactions through therapeutic drug monitoring when administering oral second-generation antipsychotics. Expert Opin Drug Metab Toxicol. 2016;12:407-422.

14. Janssen PA, Niemegeers CJ, Awouters F, et al. Pharmacology of risperidone (R 64 766), a new antipsychotic with serotonin-S2 and dopamine-D2 antagonistic properties. J Pharmacol Exp Ther. 1988;244:685-693.

15. Marder SR, Davis JM, Chouinard G. The effects of risperidone on the five dimensions of schizophrenia derived by factor analysis: combined results of the North American trials. J Clin Psychiatry. 1997;58:538-546.

16. Mannheimer B, Haslemo T, Lindh JD, et al. Risperidone and venlafaxine metabolic ratios strongly predict a CYP2D6 poor metabolizing genotype. Ther Drug Monit. 2016;38:127-134.

17. de Leon J, Susce MT, Pan RM, et al. A study of genetic (CYP2D6 and $\mathrm{ABCB} 1$ ) and environmental (drug inhibitors and inducers) variables that may influence plasma risperidone levels. Pharmacopsychiatry. 2007;40:93-102.

18. Ereshefsky L. Pharmacokinetics and drug interactions: update for new antipsychotics. J Clin Psychiatry. 1996;57(Suppl 11):12-25.

19. Corena-McLeod M. Comparative pharmacology of risperidone and paliperidone. Drugs R D. 2015;15:163-174.

20. Fang J, Bourin M, Baker GB. Metabolism of risperidone to 9-hydroxyrisperidone by human cytochromes P450 2D6 and 3A4 Naunyn-Schmiedeberg's Arch Pharmacol. 1999;359:147-151.

21. Yasui-Furukori N, Hidestrand M, Spina E, et al. Different enantioselective 9-hydroxylation of risperidone by the two human CYP2D6 and CYP3A4 enzymes. Drug Metab Dispos. 2001;29:1263-1268.

22. Xiang Q, Zhao X, Zhou Y, et al. Effect of CYP2D6, CYP3A5, and MDR1 genetic polymorphisms on the pharmacokinetics of risperidone and its active moiety. J Clin Pharmacol. 2010;50:659-666.

23. de Leon J, Bork J. Risperidone and cytochrome P450 3A. J Clin Psychiatry. 1997;58:450.

24. Bork JA, Rogers T, Wedlund PJ, et al. A pilot study on risperidone metabolism: the role of cytochromes P450 2D6 and 3A.J Clin Psychiatry. 1999;60:469-476.

25. Citrome L, Shope CB, Nolan KA, et al. Risperidone alone versus risperidone plus valproate in the treatment of patients with schizophrenia and hostility. Int Clin Psychopharmacol. 2007;22:356-362.

26. Victoroff J, Coburn K, Reeve A, et al. Pharmacological management of persistent hostility and aggression in persons with schizophrenia spectrum disorders: a systematic review. J Neuropsychiatry Clin Neurosci. 2014;26:283-312.

27. McLaughlin DB, Andrews JA, Hooper WD, et al. Apparent autoinduction of valproate beta-oxidation in humans. Br J Clin Pharmacol. 2000;49:409-415.

28. Jiang D, Bai X, Zhang Q, et al. Effects of CYP2C19 and CYP2C9 genotypes on pharmacokinetic variability of valproic acid in Chinese epileptic patients: nonlinear mixed-effect modeling. Eur J Clin Pharmacol. 2009;65:1187-1193.

29. Wen X, Wang JS, Kivisto KT, et al. In vitro evaluation of valproic acid as an inhibitor of human cytochrome $\mathrm{P} 450$ isoforms: preferential inhibition of cytochrome P450 2C9 (CYP2C9). Br J Clin Pharmacol. 2001;52: 547-553.

30. Unterecker S, Reif A, Hempel S, et al. Interaction of valproic acid and the antidepressant drugs doxepin and venlafaxine: analysis of therapeutic drug monitoring data under naturalistic conditions. Int Clin Psychopharmacol. 2014:29:206-211.

31. de Leon J. The effects of antiepileptic inducers in neuropsychopharmacology, a neglected issue. Part I: a summary of the current state for clinicians. Rev Psiquiatr Salud Ment. 2015;8:97-115.
32. Remmerie B, Ariyawansa J, De Meulder M, et al. Drug-drug interaction studies of paliperidone extended-release (ER) and divalproex sodium ER tablets in healthy participants and patients with psychiatric disorders. J Clin Pharmacol. 2016;56:683-692.

33. Nasrallah HA, Goldberg JF, Correll CU, et al. Differential diagnosis and therapeutic management of schizoaffective disorder. Ann Clin Psychiatry. 2010;22:S1-S12.

34. Staines AG, Coughtrie MW, Burchell B. N-glucuronidation of carbamazepine in human tissues is mediated by UGT2B7.J Pharmacol Exp Ther. 2004;311:1131-1137.

35. Hata M, Tanaka Y, Kyoda N, et al. An epoxidation mechanism of carbamazepine by CYP3A4. Bioorg Med Chem. 2008;16:5134-5148.

36. Magnusson MO, Dahl ML, Cederberg J, et al. Pharmacodynamics of carbamazepine-mediated induction of CYP3A4, CYP1A2, and Pgp as assessed by probe substrates midazolam, caffeine, and digoxin. Clin Pharmacol Ther. 2008;84:52-62.

37. de Leon J. The effects of antiepileptic inducers in neuropsychopharmacology, a neglected issue. Part II: pharmacological issues and further understanding. Rev Psiquiatr Salud Ment. 2015;8:167-188.

38. Yatham LN, Grossman F, Augustyns I, et al. Mood stabilisers plus risperidone or placebo in the treatment of acute mania. International, double-blind, randomised controlled trial. Br J Psychiatry. 2003;182:141-147.

39. Spina E, Avenoso A, Facciola G, et al. Plasma concentrations of risperidone and 9-hydroxyrisperidone: effect of comedication with carbamazepine or valproate. Ther Drug Monit. 2000;22:481-485.

40. Reid JG, Gitlin MJ, Altshuler LL. Lamotrigine in psychiatric disorders. J Clin Psychiatry. 2013;74:675-684.

41. Tiihonen J, Hallikainen T, Ryynanen OP, et al. Lamotrigine in treatment-resistant schizophrenia: a randomized placebo-controlled crossover trial. Biol Psychiatry. 2003;54:1241-1248.

42. Cohen AF, Land GS, Breimer DD, et al. Lamotrigine, a new anticonvulsant: pharmacokinetics in normal humans. Clinical Pharmacology \& Therapeutics. 1987;42:535-541.

43. Green MD, Bishop WP, Tephly TR. Expressed human UGT1.4 protein catalyzes the formation of quaternary ammonium-linked glucuronides. Drug Metab Dispos. 1995;23:299-302.

44. Bienentreu SD, Kronmüller KT. Increase in risperidone plasma level with lamotrigine. Am J Psychiatry. 2005;162:811-812.

45. Haen E. Therapeutic drug monitoring in pharmacovigilance and pharmacotherapy safety. Pharmacopsychiatry. 2011;44:254-258.

46. Köstlbacher A, Haen E. KONBEST - a Web-based Laboratory Information Management System (LIMS) for TDM-Laboratories. Pharmacopsychiatry. 2008;41:A23.

47. Schoretsanitis G, Stegmann B, Hiemke C, et al. Pharmacokinetic patterns of risperidone-associated adverse drug reactions. Eur J Clin Pharmacol. 2016;72:1091-1098.

48. Paulzen M, Haen E, Gründer G, et al. Pharmacokinetic considerations in the treatment of hypertension in risperidone-medicated patients - thinking of clinically relevant CYP2D6 interactions. J Psychopharmacol. 2016;30: 803-809.

49. Bader W, Melchner D, Nonenmacher T, et al. Determination of Five Commonly Used Antipsychotics in Human Serum by High Performance-Liquid Chromatography (HPLC) and Electrochemical Detection. International Meeting on Pharmacovigilance in Psychiatry, Therapeutic Drug Monitoring and Pharmacogenetics of Psychotropic Drugs; Lausanne, Switzerland, 2004. Pharmacopsychiatry. 2005;38:4.

50. Bader W. Therapeutisches Drug Monitoring bei Antipsychotika: die Entwicklung der Analytik von Antipsychotika und ihre Relevanz bei der 
Therapieleitung schizophrener Patienten am Beispiel des Arzneistoffes Risperidon: Saska, 2009, ISBN 978-3-935120-14-2.

51. Paul L, Musshoff F, Aebi B, et al. Richtlinie der GTFCh zur Qualitätssicherung bei forensisch-toxikologischen Untersuchungen. Toxichem Krimtech. 2009;76:142-176.

52. US Food and Drug Administration. Guidance for Industry on Biomedical Method Validation 2001 [Available from: http://www.fda.gov/ucm/groups fdagov-public/@fdagov-drugs-gen/documents/document/ucm070107.pdf. [Accessed 08/18/2016].

53. ICH Expert Working Group (editor) Harmonised Tripartite Guideline, Validation of Analytical Procedures: Test and Methodology. International conference on harmonisation of technical requirements for registration of pharmaceuticals for human use. Available at: http://www.ich.org/ products/guidelines/efficacy/article/efficacy-guidelines.html. Accessed September 27, 2016.

54. de Leon J. Phenoconversion and therapeutic drug monitoring. Br J Clin Pharmacol. 2015;80:777-778.

55. Schoretsanitis G, Haen E, Hiemke C, et al. Risperidone induced extrapyramidal side effects - is the need for anticholinergics the consequence of high plasma concentrations? Int Clin Psychopharmacol. 2016;31:259-264

56. Paulzen M, Haen E, Stegmann B, et al. Body mass index (BMI) but not body weight is associated with changes in the metabolism of risperidone: a pharmacokinetics-based hypothesis. Psychoneuroendocrinology. 2016;73:9-15.

57. Ono S, Mihara K, Suzuki A, et al. Significant pharmacokinetic interaction between risperidone and carbamazepine: its relationship with CYP2D6 genotypes. Psychopharmacology (Berl). 2002;162: 50-54.

58. Spina E, Scordo MG, Avenoso A, et al. Adverse drug interaction between risperidone and carbamazepine in a patient with chronic schizophrenia and deficient CYP2D6 activity. J Clin Psychopharmacol. 2001;21:108-109.
59. Takahashi H, Yoshida K, Higuchi H, et al. Development of parkinsonian symptoms after discontinuation of carbamazepine in patients concurrently treated with risperidone: two case reports. Clin Neuropharmacol. 2001;24 $358-360$.

60. Strack DK, Leckband SG, Meyer JM. Antipsychotic prescribing practices following withdrawal of concomitant carbamazepine. J Psychiatr Pract. 2009; 15:442-448.

61. Citrome L, Macher JP, Salazar DE, et al. Pharmacokinetics of aripiprazole and concomitant carbamazepine. J Clin Psychopharmacol. 2007;27: 279-283.

62. Punyawudho B, Cloyd JC, Leppik IE, et al. Characterization of the time course of carbamazepine deinduction by an enzyme turnover model. Clin Pharmacokinet. 2009;48:313-320.

63. Aichhorn W, Marksteiner J, Walch T, et al. Influence of age, gender, body weight and valproate comedication on quetiapine plasma concentrations. Int Clin Psychopharmacol. 2006;21:81-85.

64. Spina E, D'Arrigo C, Migliardi G, et al. Effect of adjunctive lamotrigine treatment on the plasma concentrations of clozapine, risperidone and olanzapine in patients with schizophrenia or bipolar disorder. Ther Drug Monit. 2006;28:599-602.

65. Andersson ML, Bjorkhem-Bergman L, Lindh JD. Possible drug-drug interaction between quetiapine and lamotrigine - evidence from a Swedish TDM database. Br J Clin Pharmacol. z2011;72:153-156.

66. Balant-Gorgia AE, Gex-Fabry M, Genet C, et al. Therapeutic drug monitoring of risperidone using a new, rapid HPLC method: reappraisal of interindividual variability factors. Ther Drug Monit. 1999;21:105-115.

67. Feng Y, Pollock BG, Coley K, et al. Population pharmacokinetic analysis for risperidone using highly sparse sampling measurements from the CATIE study. Br J Clin Pharmacol. 2008;66:629-639. 